\title{
A new concept of surgical patch used in vascular surgery
}

\author{
Natalia Lewandowska ${ }^{1, *}$ and Michał Ciałkowski ${ }^{1 \text {, }}$ \\ ${ }^{1}$ Poznań University of Technology, Chair of Thermal Engineering, Piotrowo 3, 60-965 Poznań, Poland
}

\begin{abstract}
During the surgery of atherosclerotic plaques removal, a longitudinal artery incision is made. Frequently used practice is sewing a patch in the incision area. It reduces the risk of narrowing of the artery but also causes its widening. The channel expansion leads to the enhancing separation of the boundary layer and vortexes creation. The selection of the patch size is based on the surgeon's experience. The purpose of the studies is to determine a new geometric concept of a patch. The resulting patch is fully parameterized and can be influenced by its geometric shape, adapting it to the patient's diameter. Simulations of blood flow in the artery were performed, with the patch used during the surgery and a patch developed by the authors. The analysis of the flow field in terms of the wall shear stresses (WSS) and velocity distribution has shown, that the patch developed by authors give the most satisfactory results.
\end{abstract}

\section{Nomenclature}

$\mathrm{h}(\mathrm{t})$ - Heaviside step function

$\mathrm{s}-$ width of the patch

$l-$ length of the surgical incision

$\alpha$ - the angle of the inclination

EDV - end of a diastolic velocity

EA - artery with an elliptical patch sewn in

PA - artery with a polynomial patch sewn in

PSV - peak of a systolic velocity

USG - Doppler ultrasonography

WSS - wall shear stress

\section{Introduction}

Paper present the new concept of shape of surgical patch sewn into artery during the carotid plaque removal surgery. One of the most sensitive locations where atherosclerotic plaques are deposited is the carotid artery (CA). It is a system of three vessels supplying blood to brain and facial muscles. The common carotid artery (CCA) delivers blood directly from the aorta. In the neck area, the CCA branches into two arteries: internal (ICA) and external carotid artery (ECA). The most drastic progressive stenosis occurs in the most cases in ICA [1], which is particularly dangerous because the ICA supplies the brain. Deposition of plaques in the artery is a progressive phenomenon: over time, the blockage of artery lumen (crosssectional area) increases. The divergent part of artery increases the tendency to separation of boundary layer (BL) and vortex formation. This is directly related to the viscosity of the blood, which has the highest value at the wall than in the flow core [2].

*e-mail: natalia.gr.lewandowska@ doctorate.put.poznan.pl
The vortex occurring near the wall may cause the particles to suck into the vortex and adhere to the wall. The more plaques accumulate in the artery, the narrowing (and the change of geometry) is more significant, and this increases the susceptibility to BL separation. To prevent blockage of flow in the ICA artery, the patient is subjected to regular observation. If the surface of plaque in crosssection area of the stenosed artery reaches $70 \%$ of the original arterial lumen, the patient is qualified for surgery to remove the plaque. With such advanced stenosis the possibility of the blockage of blood flow in stenosis is very high because of the occurring flow disturbances [3].

The surgery of the plaque removal requires cutting the artery and then stitching it. With extremely small diameters of the arteries (of value below $10 \mathrm{~mm}$ ), it is recommended to sew an arterial patch made of dacron, polytetrafluoroethylene - PTFE) or tissue taken from the patient (most often a vein). Patches are usually sewn in order to avoid narrowing of the artery. In extreme cases, the phenomenon of restenosis can occur: re-deposition of plaque in the location of sewing. The practice of appliance of a surgical patch reduces the risk of possible complications as a stroke, restenosis and death as compared to the primary suturing of the wound after the arteriotomy [4, 5]. The shape and length of the patch is chosen by the surgeon based on experience: the patches used often have an irregular shape, in most cases an ellipse. A badly selected patch may also lead to restenosis due to a too rapid change in the artery geometry. Currently, there are no standards and indications regarding the shape and dimensions of the patch. The article describes the concept of a standardized patch shape. 


\section{Mathematical formulation of patches}

\subsection{Mathematical formulation of patches}

Elliptical patch: Currently used patches have the shape of an ellipse. It is the easiest to cut on with scissors the surgical table. Figure 1 shows the photograph of the artery with sewn patch made during the surgery of plaque removal. The shape of the patch was reproduced by approximation with the elliptic function.

a)
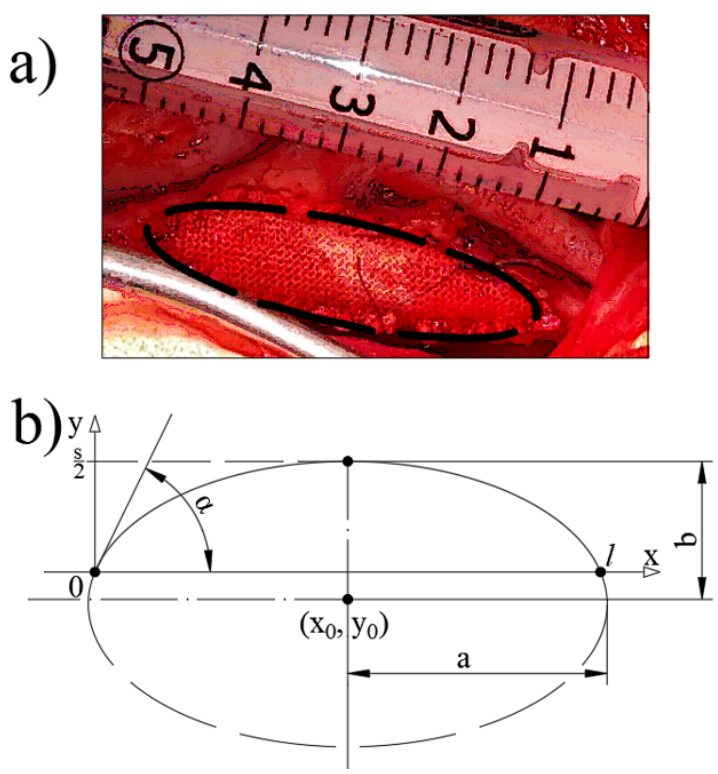

Figure 1. Shape of elliptic patch: a) a photograph b) analytical description

Input data necessary to determine the values of the function coefficient are : width of the patch (s), length of the surgical incision $(l)$, the angle of the inclination (expansion) of the deformed artery $(\alpha)$. In the function of presented parameters of the elliptical patches, the elliptic equation should be determined. Ellipse function is described by the eq. 1 :

$$
\left(\frac{\mathrm{x}-\mathrm{x}_{0}}{\mathrm{a}}\right)^{2}+\left(\frac{\mathrm{y}-\mathrm{y}_{0}}{\mathrm{~b}}\right)^{2}=1, \mathrm{~b}=\frac{\mathrm{s}}{2}-\mathrm{y}_{0}
$$

with the unknown values of parameters a and $\mathrm{y}_{0}$. It was assumed that $\alpha$ in point $\mathrm{A}(0,0)$ and $\mathrm{B}(1,0)$ has a known value. After differentiating the eq. 1 this equation goes into eq. 2 :

$$
2 \cdot \frac{x-x_{0}}{a^{2}}+2 \cdot \frac{y-y_{0}}{\left(\frac{s}{2}-y_{0}\right)^{2}} \cdot \frac{d y}{d x}=0
$$

and for point $\mathrm{A}(0,0)$ :

$$
\frac{x_{0}}{a^{2}}+\frac{y_{0}}{\left(\frac{s}{2}-y_{0}\right)^{2}} \cdot \frac{d y}{d x}=0, \frac{d y}{d x}=\tan \alpha=p
$$

therefore:

$$
\frac{x_{0}}{a^{2}}+\frac{y_{0}}{\left(\frac{s}{2}-y_{0}\right)^{2}} \cdot p=0 \rightarrow \frac{1}{a^{2}}=-\frac{x_{0}}{y_{0}} \cdot \frac{p}{\left(\frac{s}{2}-y_{0}\right)^{2}}
$$

Eq.5 was obtained while substituting the coordinates of the point $\mathrm{B}(1,0)$ to eq. 1 :

$$
\left(\frac{l-\mathrm{x}_{0}}{\mathrm{a}}\right)^{2}+\frac{\mathrm{y}_{0}^{2}}{\left(\frac{\mathrm{s}}{2}-\mathrm{y}_{0}\right)^{2}}=1
$$

and substituting eq. 1 to eq. 2 , eq. 6 was obtained.

$$
\mathrm{y}_{0}=\frac{l}{4} \cdot \frac{\mathrm{s}^{2}}{\mathrm{~s}-\frac{1}{2} \mathrm{p}}=\frac{l}{4} \cdot \frac{\left(\frac{\mathrm{s}}{l}\right)^{2}}{\frac{\mathrm{s}}{l}-\frac{\mathrm{p}}{2}}
$$

Substituting eq. 6 to eq. 4 and $x_{0}=\frac{1}{2}$ :

$$
\frac{1}{\mathrm{a}^{2}}=-\frac{\left(\frac{\mathrm{s}}{l}\right)^{2} \cdot 4\left(\frac{2 \mathrm{~s}}{\mathrm{p} \cdot l}-1\right)}{l^{2}\left(\frac{2 \mathrm{~s}^{2}}{\mathrm{p} \cdot l}-\frac{\mathrm{s}}{l}\right)}
$$

when $\mathrm{p} \rightarrow \infty, \alpha=\frac{\pi}{2}$. Therefore $\mathrm{a}=\frac{1}{2}$. Finally:

$$
\begin{gathered}
\mathrm{y}=\mathrm{y}_{0}+\left(\frac{\mathrm{s}}{2}-\mathrm{y}_{0}\right) \cdot \sqrt{1-\left(\frac{\mathrm{x}-\mathrm{x}_{0}}{\mathrm{a}}\right)^{2}} \\
\mathrm{y}_{0}=\frac{1}{4} \cdot \frac{\left(\frac{\mathrm{s}}{l}\right)^{2}}{\frac{\mathrm{s}}{l}-\frac{\mathrm{p}}{2}}, \mathrm{x}_{0}=\frac{l}{2} \\
\frac{1}{\mathrm{a}^{2}}=-\frac{1}{2}\left(\frac{\mathrm{s}}{l}\right) \cdot \frac{\mathrm{p} \cdot\left(\frac{\mathrm{s}}{l}-\frac{\mathrm{p}}{2}\right)}{l^{2}\left(\frac{1}{2}\left(\frac{\mathrm{s}}{2}\right)^{2}-\frac{\mathrm{s}}{l} \cdot \frac{\mathrm{r}_{\text {art }}}{4}\right)^{2}}
\end{gathered}
$$

Polynomial patch: The contour of the polynomial patch providing a continuous change in the artery crosssectional area should fulfill the following conditions:

- contact of the patch contour at the beginning and end of sewing with the primary artery,

- symmetry with respect to the $\mathrm{x}$-axis (center point) and $y$-axis. The outline of the patch is shown in fig. 2.

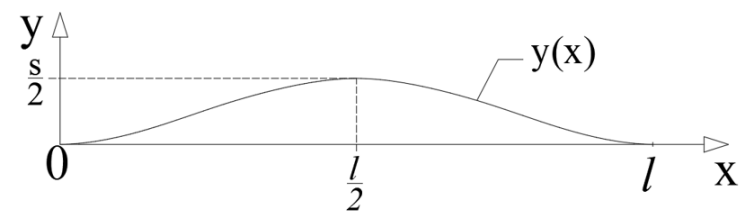

Figure 2. Function of polynomial patch

It was assumed that the patch width is maximum value occurring in the middle point. Polynomial function is described by the eq. 11 :

$$
y(x)=a_{0}+a_{1} x+a_{2} x^{2}+a_{3} x^{3}+a_{4} x^{4}
$$

Therefore, according to figure 2, there can be obtained the following conditions

- zeroing of the function $\mathrm{y}(\mathrm{x})$ at points $\mathrm{x}=0$ and $\mathrm{x}=l$ :

$$
\begin{gathered}
\mathrm{y}(0)=\mathrm{a}_{0}+\mathrm{a}_{1} 0+\mathrm{a}_{2} 0^{2}+\mathrm{a}_{3} 0^{3}+\mathrm{a}_{4} 0^{4}=0 \rightarrow \mathrm{a}_{0}=0 \\
\mathrm{y}(l)=\mathrm{a}_{0}+\mathrm{a}_{1} l+\mathrm{a}_{2} l^{2}+\mathrm{a}_{3} l^{3}+\mathrm{a}_{4} l^{4}=0
\end{gathered}
$$


- in the middle point:

$$
\mathrm{a}_{1}+\mathrm{a}_{2}\left(\frac{l}{2}\right)+\mathrm{a}_{3}\left(\frac{l}{2}\right)^{2}+\mathrm{a}_{4}\left(\frac{l}{2}\right)^{3}=\frac{\mathrm{s}}{l}
$$

- tangential transition into the $\mathrm{x}$-axis in the point $\mathrm{x}=0$ and $\mathrm{x}=1$

$$
\begin{gathered}
\mathrm{y}^{\prime}(0)=0 \rightarrow \mathrm{a}_{1}=0 \\
\mathrm{y}^{\prime}(\mathrm{l})=\mathrm{a}_{1}+2 \mathrm{a}_{2} l+3 \mathrm{a}_{3} l^{2}+4 \mathrm{a}_{4} l^{3}=0
\end{gathered}
$$

Based on eq. 12-16 the system of equation was obtained. The solution gives a function coefficient dependent on geometrical parameters of artery and the patch:

$$
\begin{gathered}
\mathrm{a}_{0}=\mathrm{a}_{1}=0, \mathrm{a}_{2}=8 \frac{\mathrm{s}}{l^{2}}, \mathrm{a}_{3}=-16 \frac{\mathrm{s}}{l^{3}}, \mathrm{a}_{4}=8 \frac{\mathrm{s}}{l^{4}} \\
\mathrm{y}(\mathrm{x})=8 \mathrm{~s}\left(\frac{\mathrm{x}}{l}\right)^{2}\left(\frac{\mathrm{x}}{l}-1\right)^{2}
\end{gathered}
$$

\subsection{Numerical study}

Artery geometrical model: Internal carotid artery (ICA) with a sewn elliptical and polynomial patch was modelled as straight-forward channel (Fig. 3). ICA diameter is equal to $10 \mathrm{~mm}$. Length and maximum width of patches have the same value. Width of the patch is equal to $9 \mathrm{~mm}$, what causes the increase in diameter up to value $6.5 \mathrm{~mm}$ in the widest part of the artery. It was assumed, that the expansion of the artery is symmetric. Length of the patch was set to $45 \mathrm{~mm}$. The computational grid created for the purpose of the blood flow simulation was a structured multiblock mesh with implemented inflation layers $(\mathrm{y}+=$ 1) and $\mathrm{OH}$ topology. Mesh contains about 250 thousand elements.

a)

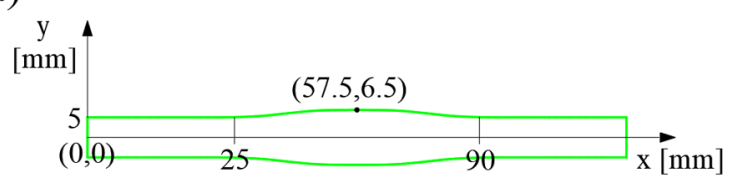

b)

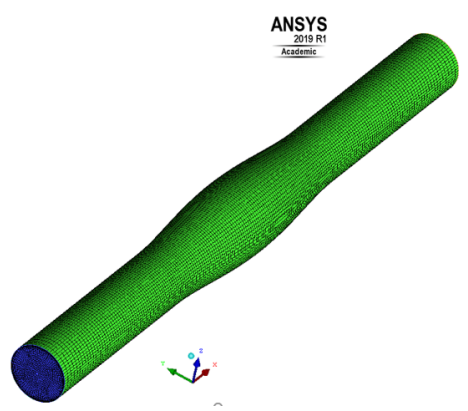

Figure 3. Modified artery model with sewn polynomial patch

Boundary conditions: At the inlet a boundary condition of the velocity inlet type was assumed. The blood flow velocity in the artery is cyclic variable over time, depending on the heart rate. Based on USG examination
Table 1. Velocity function coefficients

\begin{tabular}{cccccc}
\hline $\mathrm{i}$ & 1 & 2 & 3 & 4 & 5 \\
\hline $\mathrm{a}_{\mathrm{i} 0}$ & -34 & -154.5 & 711.8 & 449 & -0.086 \\
$\mathrm{a}_{\mathrm{i} 1}$ & 6.8 & 55.8 & -989.3 & -600 & 0.26 \\
$\mathrm{a}_{\mathrm{i} 2}$ & 0.19 & -6.5 & 509.9 & 265.6 & 0.0 \\
$\mathrm{a}_{\mathrm{i} 3}$ & 0.0 & 0.78 & -115.4 & -38.7 & 0.0 \\
$\mathrm{a}_{\mathrm{i} 4}$ & 0.0 & 0.0 & 9.98 & 0.0 & 0.0 \\
\hline $\mathrm{t}_{\mathrm{i} 1}$ & 0.0 & 0.1 & 0.22 & 0.41 & 0.48 \\
$\mathrm{t}_{\mathrm{i} 2}$ & 0.1 & 0.22 & 0.41 & 0.48 & 0.83 \\
\hline
\end{tabular}

results for ICA performed on a healthy patient, the transient velocity function was approximated with 5 polynomial spline functions. The velocity inlet function is described by the eq. 19 :

$$
u(t)=\sum_{i=1}^{5} \sum_{j=0}^{5} a_{i j} t^{j}\left[h\left(t-t_{i 1}\right)-h\left(t-t_{i 2}\right)\right]
$$

Function coefficient are given in table 1 . Values of $a_{i j}$ was determined based on PSV, EDV, S/D and HR obtained from USG. Figure 4 shows the comparison between the real and approximated value. At the out-

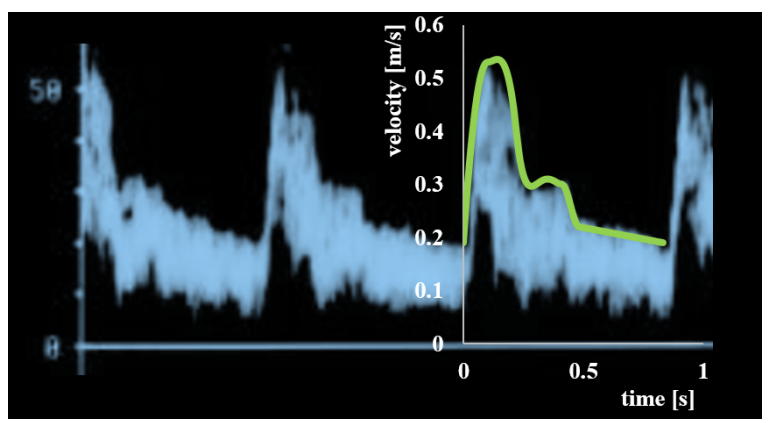

Figure 4. Velocity time function obtained from USG

flow from the artery, pressure-outlet boundary condition was applied. Static pressure was implemented as timevarying pulse function described by 2-parameter Windkessel model $[6,7]$. Walls of the artery model was set as stiff with no-slip boundary condition.

Fluid model: blood is non-Newtonian liquid. It depends on the shear rate: one of the most popular blood viscosity model is Carreau-Yasuda [8, 9], which was included in considered cases as a fluid viscosity model.

Numerical setup: calculations were carried out with the use of Ansys Fluent software. Due to the blood flow is incompressible and Reynolds number doesn't exceeds the value of 5000, it was decided to use the pressure-based solver. k- $\omega$ SST with low-Re corrections [10] was applied as a turbulence model. The time step was set to value 0.001 . The simulated flow time was equal to $0.83 \mathrm{~s}$, which corresponds to 830 time steps. For the method of solving the governing equations, coupled solver was chosen. All equation was solved on second order of discretization, to increase the accuracy of the simulation results[11]. 


\section{Results and discussion}

The numerical research results analysis was performed based on two flow parameters: velocity and wall shear stresses (WSS). It's important to determine how the shape of patch impact on the WSS values, because locally increased values may lead to the weakening of artery wall, what may result in aneurysm formation. Area-weighted WSS was taken into consideration. It was calculated with the use of formula:

$$
\Phi_{\text {ave }}=\frac{1}{\mathrm{~A}} \oiiint_{\mathrm{A}} \Phi \mathrm{d} \mathrm{A}=\sum \Phi_{\mathrm{i}} \mathrm{A}_{\mathrm{i}}
$$

where $\Phi$ is considered physical variable, $\Phi_{i}$ is value of WSS in the mesh element $A_{i}$, and $A$ is the total surface on the wall calculated between the $\mathrm{x}=25 \mathrm{~mm}$ and $\mathrm{x}=90$ mm. Maximum value of WSS was also taken into considerations: figure 5 presents those value as a function of pulse time.

The average values of WSS are similar. The most significant differences can be observed when considering the $\mathrm{WSS}_{\max }$ values occurring during cardiac cycle. The $\mathrm{WSS}_{\max }$ for an artery with an elliptical patch sewn in (EA) is $12 \mathrm{~Pa}$ and is twice as high as the WSS values for an artery with a polynomial patch (PA). That may indicate high irregularity with the WSS distribution on the wall. Figures 6-8 show the distribution of stresses on the wall

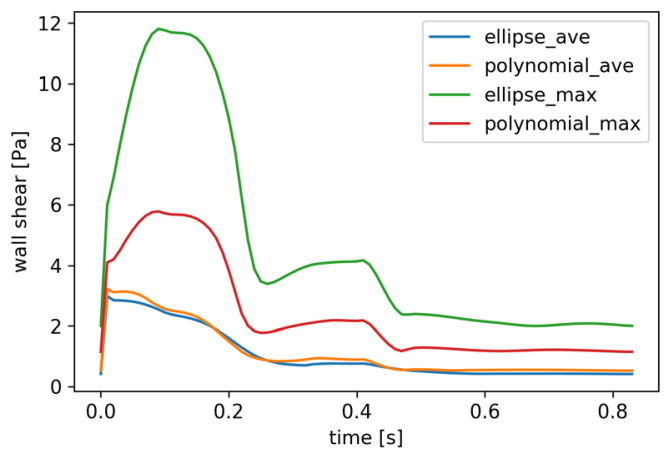

Figure 5. Area-weighted average and maximum value of WSS during one cardiac cycle

in the area of local artery dilatation. The analysis includes three characteristic cardiac cycle stages: peak of the systolic phase (PSV, $\mathrm{t}=0.14 \mathrm{~s}$ ), transition time between systolic and diastolic phase $(\mathrm{t}=0.30 \mathrm{~s})$ and end of diastolic phase $(E D V, t=0.80 \mathrm{~s})$. Due to substantial change in WSS during pulse time, the legend was scale to the range of WSSmax the considered timestamp. When comparing the two distributions with each other, it can be seen that in the PA, variation of WSS in the convergent part of the artery is distributed over a more sizeable part of the surface due to the smooth course of the geometry. For EA, it can be observed a concentrated increase in WSS when passing between deformed geometry and the primary artery: the change in the cross-section area of the artery along the $\mathrm{x}$ axis is much more severe than in the polynomial patch, which results in the aforementioned jump in WSS value. This tendency can be observed in all of the time steps considered. The local extreme values of WSS strongly depends on artery geometry: the more raping change in the shape of the artery more concentrated WSS distribution. Considering that areas of concentrated stresses may develop aneurysm $[12,13]$, and plaque deposition [14] the polynomial patch is much more beneficial because it provides for less rapid change in geometry. One of the most

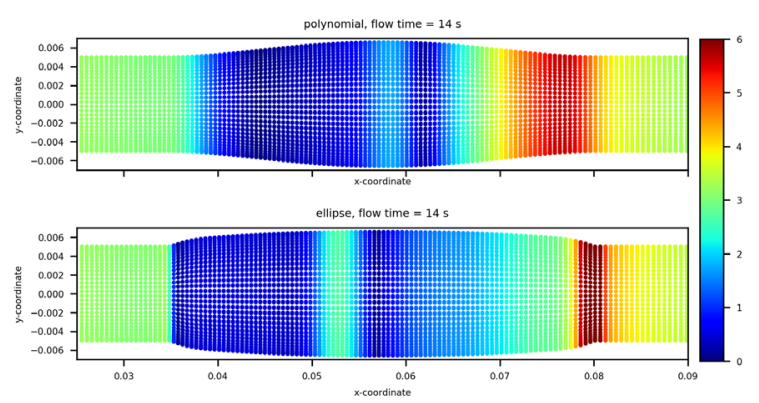

Figure 6. WSS distribution, $t=0.14 \mathrm{~s}$

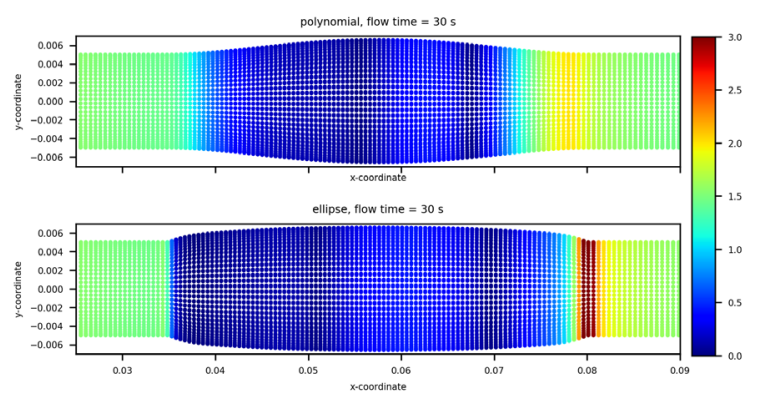

Figure 7. WSS distribution, $\mathrm{t}=0.30 \mathrm{~s}$

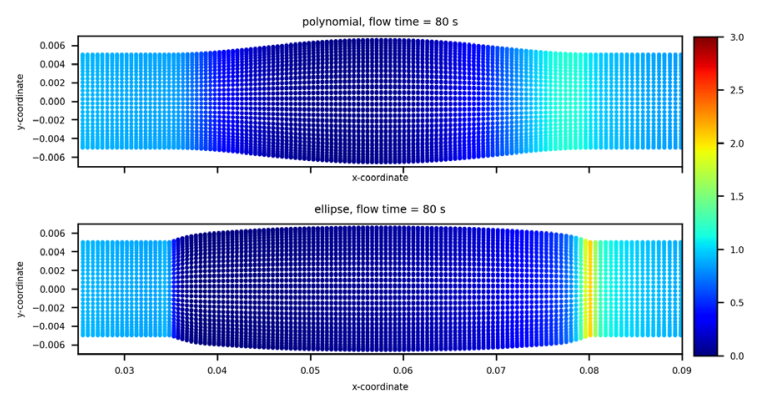

Figure 8. WSS distribution, $\mathrm{t}=0.80 \mathrm{~s}$

important reasons for using polynomial patches is reduction of the areas in which the boundary layer has been separated. This minimizes the risk of the restenosis due to the fact that in the regions of separated flow often a recapture of atherosclerotic plaques occurs. Thanks to the application of polynomial patches, these areas can be significantly reduced. This tendency can be observed in Figures 9-11, where the velocity distribution in the axial section of the 
examined arteries is presented. In Figure 9, it can also be seen that the change in velocity in EA is more rapid comparing with PA. High velocity gradient increases the risk of occurring the flow disturbances.

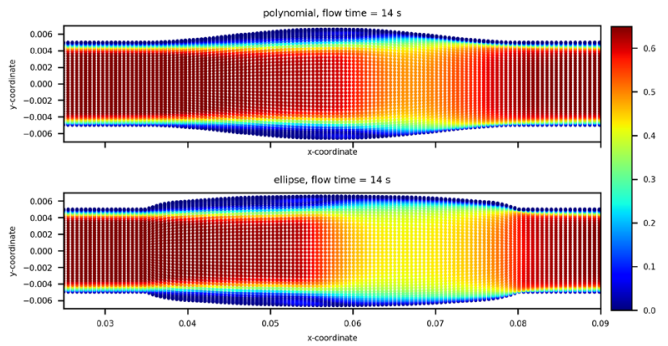

Figure 9. Velocity distribution in axial section, $\mathrm{t}=0.14 \mathrm{~s}$

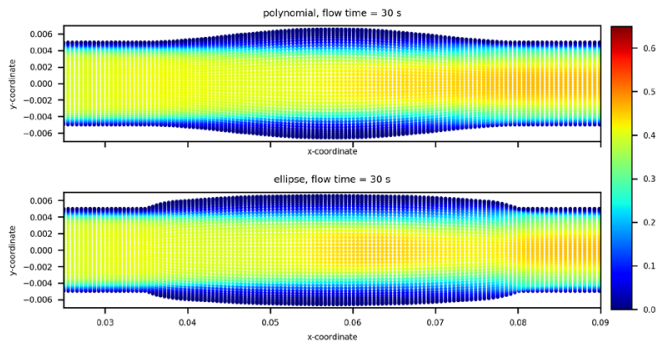

Figure 10. Velocity distribution in axial section, $t=0.30 \mathrm{~s}$

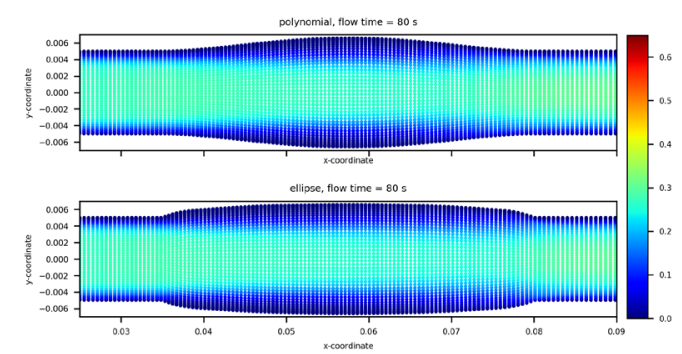

Figure 11. Velocity distribution in axial section, $t=0.80 \mathrm{~s}$

\section{Conclusions}

The application of polynomial patches instead of elliptic ones results in the reduction of WSS, which minimizes the risk of a local wall degeneration and aneurysm formation. This tendency is most noticeable at the convergent section of the deformed artery where the increase of WSS in EA is twice as high. Therefore, it was significantly more concentrated. The resulting patch is fully parameterized: its shape can be adjusted to the patient's artery diameter, length of the surgical incision and desired patch width. Fully parameterized patch model allows to develop a group of standardized patches ready for mass production. As consequence, the vascular surgeon, instead of cutting the patch during the operation, will have a ready set of patches. Patch's width and length can be adjusted to the patient's diameter and the length of the artery incision. Such a practice, in addition to minimizing the risk of restenosis, will also shorten the time of surgery by the time needed to precisely cut an elliptical patch.

\section{Acknowledgements}

This research was supported in part by PLGrid Infrastructure.

\section{References}

[1] C. Liapis, S.P. Bell, D. Mikhailidis, J. Sivenius, A. Nicolaides, J. Fernandes e Fernandes, G. Biasi, L. Norgren, Eur. J. Vasc. Endovasc. Surg. 37, 1 (2009)

[2] A.J. Apostolidis, A.P. Moyer, A.N. Beris, J. Nonnewton. Fluid Mech. (2015)

[3] R. Tabe, F. Ghalichi, S. Hossainpour, K. Ghasemzadeh, Biomed. Mater. Eng. 27, 119 (2016)

[4] A. Muto, T. Nishibe, H. Dardik, A. Dardik, J. Vasc. Surg. 50, 206 (2009)

[5] K. Rerkasem, P.M. Rothwell, Cochrane Database Syst. Rev. p. CD000160 (2009)

[6] N. Westerhof, J.W. Lankhaar, B.E. Westerhof, Med. Biol. Eng. Comput. 47, 131 (2009)

[7] M. Catanho, M. Sinha, V. Vijayan, BENG 221Mathematical Methods in Bioengineering Model of Aortic Blood Flow Using the Windkessel Eect (2012), pp. 1-15, http://www.beltina.org/ health-dictionary/

[8] R.B. Bird, R.C. Armstrong, O. Hassager, John Wily sons Inc (1987)

[9] Y.I. Cho, K.R. Kensey, Biorheology 28, 241 (1991)

[10] F.R. Menter, Int. J. Comut. Fluid Dyn. (2009)

[11] ANSYS-Fluent User's Guide, Release 18.2 (2015)

[12] Y.Q. Zhu, M.H. Li, L. Yan, H.Q. Tan, Y.S. Cheng, J. Neuropathol. Exp. Neurol. 73, 808 (2014)

[13] S. Omodaka, T. Inoue, K. Funamoto, S.I. Sugiyama, H. Shimizu, T. Hayase, A. Takahashi, T. Tominaga, J. Biomech. 45, 2355 (2012)

[14] H.C. Groen, . Frank, J.H. Gijsen, . Aad Van Der Lugt, M.S. Ferguson, T.S. Hatsukami, A.F.W. Van Der Steen, C. Yuan, J.J. Wentzel (2007) 